\title{
Uso de íris digitalizada na reabilitação protética ocular
}

\author{
Use of digital iris in ocular prosthetic rehabilitation
}

Ricardo Cesar dos Reis*

Reinaldo Brito e Dias ${ }^{* *}$

\section{Resumo}

Introdução: um dos fatores imprescindíveis no resultado estético satisfatório do tratamento reabilitador protético ocular é a reprodução fiel da íris do olho remanescente na íris protética. Trata-se da dissimulação da perda para os pacientes afetados. Objetivo: este trabaIho apresenta uma nova técnica de confecção da íris protética, utilizando imagem fotográfica digitalizada da íris remanescente, em adesivo. Métodos: em razão da fotodegradação que compromete a longevidade das próteses oculares, decidiu-se avaliar a estabilidade das cores, da técnica proposta, por meio de teste de enveIhecimento acelerado. Para os ensaios, foram confeccionados corpos de prova simulando a íris protética, reproduzida em adesivo, nas cores verde, azul, marrom e preto. A variação do grau de degradação foi avaliada mediante leitura colorimétrica inicial e após, em três intervalos semanais, durante o envelhecimento artificial com radiação ultravioleta. Resultados: a análise estatística sugere que, quando as cores foram avaliadas em função do período de ensaio de envelhecimento artificial, somente na cor azul houve diferença estatisticamente significativa. Conclusões: nenhuma das cores apresentou uma degradação de cor acima do nível clinicamente aceitável, resultado bastante favorável para a viabilidade da técnica proposta.

Palavras-chave: Olho artificial. Íris. Cor.

\section{Introdução}

Os distúrbios estéticos e funcionais provocados pela perda ocular são amenizados pela reabilitação protética ocular. Os olhos são a região de identificação do indivíduo e o foco principal das relações interpessoais. Em razão disso, esse comprometimento estético desencadeia transtornos emocionais, limitando as atividades cotidianas do indivíduo ${ }^{1}$. Funcionalmente, a importância da prótese ocular é diversa. Ela protege a sensível mucosa que reveste a cavidade, previne o colapso e a deformação das pálpebras, possibilita o direcionamento da drenagem lacrimal e a manutenção do tônus muscular, evitando assimetria no contorno facial ${ }^{2}$. A reabilitação da íris na prótese ocular deve ser fiel, uma vez que os olhos são um órgão par, sendo fundamental no resultado estético e na satisfação da reabilitação.

Os diversos trabalhos propondo diferentes técnicas de confecção de íris ratificam a preocupação dos autores com a fidelidade na reprodução da íris protética, utilizando vários tipos de papéis e tintas para a confecção do botão da íris ${ }^{3}$. Outro aspecto de constantes estudos é a durabilidade da prótese ocular, provocada pela fotodegradação das cores através da radiação solar ${ }^{4}$. Essa degradação limita a vida útil da prótese, consequentemente, há necessidade de sucessivas trocas, o que se torna uma dificuldade para os pacientes de localidades distantes e aumenta a demanda no atendimento.

Por meio de ensaios com envelhecimento acelerado e avaliação colorimétrica, tem-se demonstrado a instabilidade de algumas cores sobre a vida útil dos diversos pigmentos utilizados na pintura, entre outras técnicas aplicadas.

Especialista em Prótese Bucomaxilofacial pela Associação Brasileira de Ensino Odontológico (Abeno), São Paulo, SP, mestre e doutor pela Faculdade de Odontologia da Universidade de São Paulo, São Paulo, SP, Brasil.

Professor titular em Prótese Bucomaxilofacial e chefe do Departamento de Cirurgia, Prótese e Traumatologia Bucomaxilofaciais da Faculdade de Odontologia da Universidade de São Paulo, São Paulo, SP, Brasil. 
O aperfeiçoamento e o acesso à fotografia e aos programas digitais possibilitaram a reprodução de imagens com mais fidelidade e qualidade estética. A primeira técnica proposta, que utilizava a imagem digitalizada da íris remanescente obtida por fotografia digital, impressa em papel com jato de tinta, apresentou resultados estéticos bastante favoráveis, porém, sem oferecer comprovação quanto à longevidade ${ }^{5,6,7}$. Em outra técnica, a imagem digital da íris foi revelada em papel fotográfico e colada na base e na calota acrílica incolor, sendo comparada à técnica tradicional de pintura com tinta acrílica. Foram obtidos resultados favoráveis e semelhantes quanto à degradação das cores entre as técnicas, mostrando nova alternativa na confecção da prótese digital ${ }^{6,8,9,10}$.

Este trabalho propõe uma nova técnica de obtenção da íris protética por meio da imagem digitalizada da íris remanescente impressa em adesivo, além do estudo da estabilidade das cores sob a ação da radiação para a técnica proposta. Pode-se obter uma reprodução fiel na confecção da íris protética, imprescindível na estética geral da prótese ocular.

\section{Revisão da literatura}

\section{Prótese ocular}

Murphey et al. ${ }^{10}$ (1945) estão entre os primeiros autores que utilizaram fotografias do olho remanescente determinando o diâmetro exato da íris e pupila. Desenhavam, em papel liso especial para aquarela, uma circunferência ligeiramente menor que a íris natural, compensando o efeito de lente causado pela resina acrílica incolor.

Rezende et al. ${ }^{2}$ (1997), por sua vez, apresentam a técnica de pintura com tinta acrílica diretamente na calota acrílica, eliminado a etapa da pintura em papel ou cartolina. Uma das vantagens foi a possibilidade de avaliar-se a cor e a tonalidade com o efeito do acrílico da calota convexa. A técnica proposta oferece vantagens em tempo de confecção e de manuseamento do material.

Artopolou et al. ${ }^{5}$ (2006) apresentaram uma técnica de obtenção da íris protética, utilizando imagem digital mediante impressão com jato de tinta. Os autores indicaram o equipamento utilizado para a obtenção da imagem, mencionando a utilização de recursos digitais e descrevendo a técnica de montagem da íris protética. Concluíram ser uma técnica que reduz o tempo de confecção e que apresenta resultado estético satisfatório, porém, salientaram que estudos complementares são importantes para comprovar sua longevidade, principalmente no que se refere ao fator fotodegradação.

Reis $^{6}$, em 2008, destacou a importância da fiel reprodução da íris na prótese ocular e apresentou uma nova técnica de confecção da íris protética, substituindo a pintura tradicional pela imagem digitalizada da íris remanescente revelada em papel fotográfico. Decidiu-se avaliar a estabilidade das cores, comparando-se a técnica de pintura com a obtida pela fotografia digital, sob a ação de dois fatores: ação térmica do ciclo de polimerização da resina acrílica e o ensaio de envelhecimento.

Jain et al. ${ }^{9}$ (2010) descreveram um caso clínico de reabilitação protética usando imagem digital da íris do paciente. A fotografia foi comparada com a íris natural do paciente. A imagem digital proporciona resultados estéticos aceitáveis, uma vez que permite réplicas da íris do paciente com o mínimo de ajustes de cor e modificações. O método é simples, menos demorado e requer um mínimo de competências artísticas, que são necessários na técnica da pintura da íris.

Jayaswal et al. ${ }^{11}$ (2011) destacaram que a íris pode ser feita por pintura ocular ou pela fotografia digital. Os melhores resultados cosméticos e funcionais de uma prótese individualizada ampliaram a satisfação do paciente, proporcionando-o um estilo de vida normal.

Sidharth et al. ${ }^{12}$ (2010) mencionaram que cabe ao protesiólogo maxilofacial a fabricação de uma prótese ocular essencial com estética aceitável e que restaure a aparência normal. Os autores apresentaram uma técnica de impressão simplificada e confecção da prótese ocular, utilizando a fotografia digital da íris do paciente, para fins comparativos. Ajustes no brilho, na cor, no contraste, na cor da imagem foram feitos por meio de softwares gráficos.

Kale et al. ${ }^{13}$, em 2008, apresentaram uma técnica para a fabricação de uma prótese ocular, usando tecnologia de imagem digital. A técnica descrita fornece uma escolha de baixo custo e serve como um auxiliar de diagnóstico para predizer a conformidade do paciente ao tratamento protético ocular. Apresentou vantagens estéticas e facilidade relativa de fabricação da prótese ocular.

Goulart et al. ${ }^{1}$ (2011) ressaltou que a perda de um globo ocular tem impacto psicológico, exigindo adaptação. Intervenções como a reparação de próteses parecem levar a uma mudança emocional e comportamental. Os dados mostraram dificuldades emocionais no contato inicial com a perda de um olho e o papel da prótese como um elemento de inclusão social, sublinhando a importância do trabalho em equipe e do acesso aos serviços de montagem na adaptação desses pacientes.

\section{Envelhecimento artificial acelerado}

Brenan e Fedor ${ }^{4}$ (1994) enfatizaram que o comportamento de um material em determinadas condições, por meio dos testes de envelhecimen- 
to artificial, é amplamente observado em diversas pesquisas para o desenvolvimento e o controle da qualidade de distintos materiais. A degradação fotoquímica ocorre por quebra de ligações químicas do substrato, causadas pelos fótons de luz, variando pela suscetibilidade de cada tipo de composição química a um determinado comprimento de onda.

Reis et al. ${ }^{14}$ (2008) avaliaram a estabilidade de cor de tintas acrílicas no que diz respeito a dois fatores: ciclo de polimerização durante a fabricação da prótese ocular e envelhecimento artificial acelerado. Foi realizada uma leitura espectofotométrica antes e depois da polimerização das amostras, as quais, posteriormente, foram submetidas ao envelhecimento acelerado durante três semanas, com leituras colorimétricas semanais. Após a ação do ciclo de polimerização, apenas a cor amarela ficou um pouco acima do nível clinicamente aceitável de delta E. Após o envelhecimento acelerado, houve diferença estatística para as cores verde, marrom, preto e azul. As cores marrom e preta ficaram abaixo do nível clinicamente aceitável, as cores verde e amarela ficaram ligeiramente acima e a cor azul ficou bastante elevada. Concluíram os autores que apenas na cor amarela ocorreu pequena alteração de cor após a ação do ciclo de polimerização.

D'Almeida $^{15}$, em 1999, avaliou a estabilidade de cor das tintas acrílica hidrossolúvel e automotiva à base de laca acrílica nas cores preta, marrom, azul e branca. Os corpos de prova (CPs) foram submetidos ao ensaio de envelhecimento acelerado e leituras colorimétricas. De acordo com os resultados obtidos, em ambas as tintas ocorreu alteração de cor, em aspectos semelhantes com alta degradação para a cor azul, seguida da cor branca. As cores marrom e preta apresentaram ótima estabilidade quando expostas à radiação ultravioleta $\mathrm{B}$.

Goiato et al. ${ }^{16}$ (2011) avaliaram a estabilidade de cor de íris artificiais obtidas com duas técnicas (pintura a óleo e imagem digital) nas cores marrom e azul e submetidas à polimerização por micro-ondas. As alterações cromáticas ocorridas em todas as amostras apresentaram diferenças estatisticamente significativas entre as técnicas avaliadas. Para a cor marrom, na técnica digital, a diferença foi de 13,63, e para a cor azul, de 16,68. Na técnica de pintura com tinta a óleo, houve índice de 21,10 de degradação para a cor marrom e de 20,23 para a cor azul. Independentemente da técnica, todas as amostras sofreram alteração de cor após polimerização por micro-ondas.

Fernandes et al. ${ }^{17}$ (2009) avaliaram a estabilidade de cor de tintas utilizadas na pintura de íris em próteses oculares, incluindo tinta acrílica hidrossolúvel, automotiva à base de nitrocelulose, guache hidrossolúvel e a óleo, polimerizadas por energia de micro-ondas, variando-se a tinta, o método de secagem e o período de envelhecimento acelerado. Os CPs foram submetidos a uma câmara de envelhecimento acelerado, sob a radiação ultravioleta, por 1.008 horas. Todas as tintas apresentaram altera- ção cromática. A tinta a óleo apresentou a maior resistência diante do envelhecimento acelerado, independentemente do método de secagem e do período de envelhecimento.

Kabawata $^{18}$ (2006) avaliou a estabilidade de cores dos pigmentos utilizados na caracterização da esclera em prótese ocular. Os CPs foram divididos em dois grupos, resina de esclera tipo 1 e tipo 2. Após o envelhecimento artificial e a leitura com espectofotômetro, concluiu que a estabilidade depende do tipo de resina e dos pigmentos extrínsecos empregados na caracterização. Os três pigmentos azuis, em ambas as resinas, apresentaram uma estabilidade muito aquém do limite clinicamente aceitável.

Mundim et al. ${ }^{19}$ (2011) avaliaram quatro tipos de tintas, guache, óleo, acrílica e resina, para a caracterização de esclera nas cores azul e marrom submetidos ao envelhecimento artificial acelerado. Os resultados demonstraram que a cor marrom apresentou as médias inferiores de $\Delta \mathrm{E}^{*}$ comparadas à cor azul. Os autores concluíram que apenas o grupo tinta acrílica para o pigmento marrom mostrou valores clinicamente aceitáveis para a estabilidade de cor após envelhecimento artificial acelerado.

Tendo em vista o referencial teórico exposto, o presente estudo objetiva o desenvolvimento e avaliação colorimétrica de nova técnica de confecção de íris em prótese ocular obtida através da impressão da imagem digitalizada da íris do olho remanescente em adesivo.

\section{Métodos}

Para obter-se a imagem total da esfera da íris, a fotografia digital é realizada com abertura palpebral que possibilite a completa visualização e captação de imagem da íris (Figura 1). A máquina fotográfica digital utilizada foi Sony Cyber-Shot DSC-H2®. As fotos são obtidas no modo macro, com distância e foco seguindo as especificações técnicas ${ }^{7}$.

A imagem é transferida da máquina fotográfica para o computador LG $^{\circledast}$ (LG Eletronics, Manaus, Brasil), e, utilizando-se o Adobe Photoshop 7.0 ${ }^{\circledR}$, por meio dos recursos de alteração, são feitos ajustes, retirando-se os reflexos e definindo-se o diâmetro da íris e de suas estruturas anatômicas. Posteriormente, é feita a montagem em arquivo JPEG no formato para impressão com diferentes imagens obtidas (Figura 1 ).

A impressão digital no adesivo é feita em uma máquina específica MIMAK ${ }^{\circledR}$ (Japão) na empresa RTB - Impressão Digital (Rio Claro-SP), que usa tinta à base de solvente. $\mathrm{O}$ adesivo usado na impressão é $3 \mathrm{M}$, transparente, modelo $\mathrm{D} 600^{\circledR}$. Posteriormente à sua impressão, o adesivo é afixado na placa acrílica leitosa com auxílio de uma espátula de plástico de maneira homogênea, a fim de evitar a formação de bolhas. 


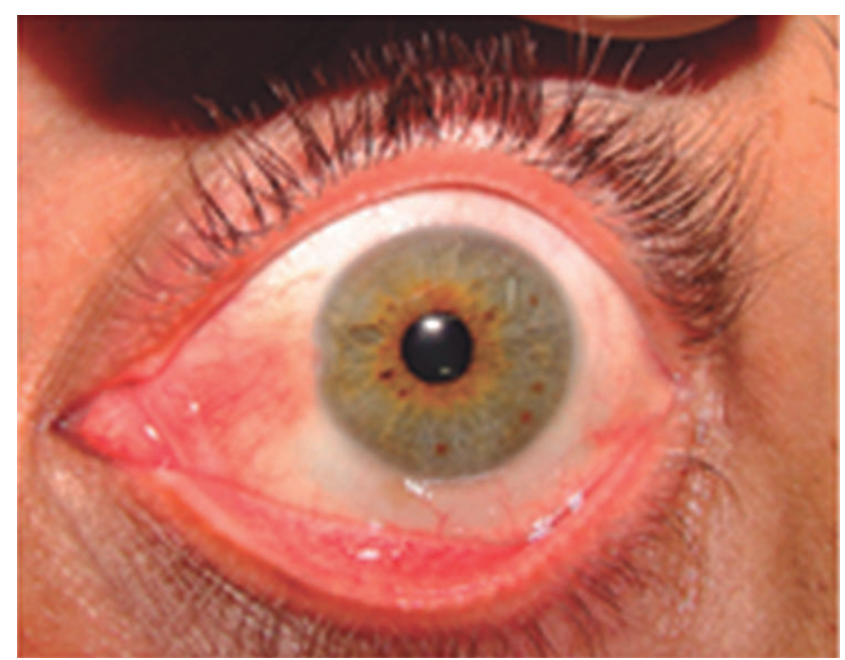

Figura 1 - Fotografia com a abertura palpebral do olho contralateral

A imagem colada nessa placa substitui a colagem na base acrílica branca na montagem tradicional da íris protética. Para finalizar a montagem do botão da íris, cola-se manualmente sobre a imagem a calota acrílica incolor convexa, e, depois do acabamento, a imagem deve ficar isolada entre as peças em resina. Na sequência, procede-se à colagem na junção de ambas com Super Bonder ${ }^{\circledR}$ para evitar o contato com os componentes químicos da polimerização que afetam a imagem ${ }^{6}$ (Figura 2 ).

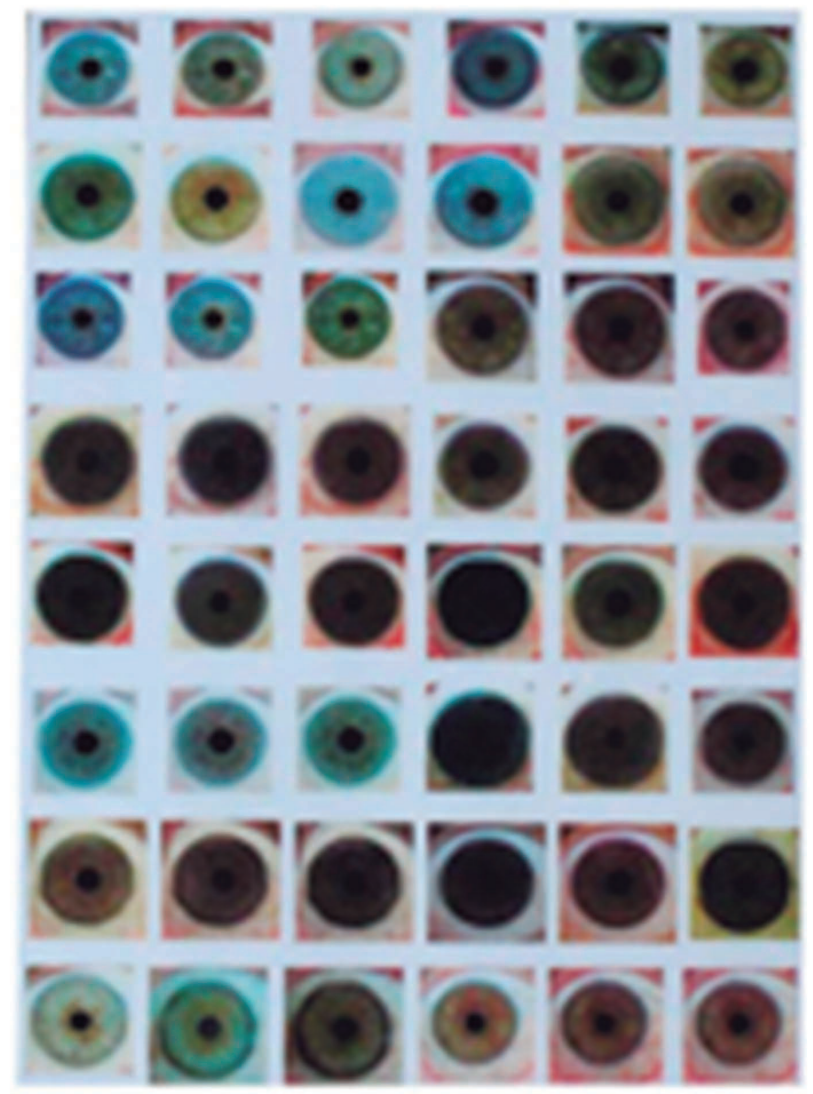

Figura 2 - Íris impressas na etiqueta autocolante plastificada
Na presença do paciente, é escolhida a imagem que ficou mais semelhante à íris remanescente, procedendo-se ao seu recorte, no limite do seu entorno, com pedras de desgaste para peça de baixa rotação.

\section{Obtenção dos corpos-de-prova}

Foram obtidos 24 CPs para a técnica da impressão em adesivo, sendo $6 \mathrm{CPs}$ para cada cor: verde, azul, marrom e preto.

Para a obtenção de fotografias com cores uniformes e monocromáticas, utilizou-se a escala de cores do programa digital Power Point $₫$, por meio do preenchimento do plano de fundo do slide, salvando em formato JPEG. Optou-se pelas cores da escala desse programa mais próximas às cores utilizadas na pintura com tinta acrílica ${ }^{6,7}$.

Placas de acrílico de $2 \mathrm{~mm}$ de espessura nas cores branco leitoso e transparente foram cortadas em uma máquina de recorte com fresa AXYS Itália. Para o ensaio, 24 placas de acrílico incolor e 24 placas brancas leitosas foram recortadas, seguindo as especificações do suporte interno da máquina de envelhecimento artificial nas medidas $7,5 \times 15 \mathrm{~cm}$.

A imagem das cores foi enviada para a mesma empresa que realizou a impressão das íris, na qual são feitas a impressão e a montagem do adesivo nas cores especificadas na placa acrílica branca. Posteriormente, fez-se a colagem da placa acrílica incolor com adesivo instantâneo (Super Bonder ${ }^{\circledR}$ ), que também é utilizada na junção entre as duas placas acrílicas. Para finalizar a montagem, como no grupo adesivo, foi realizada a vedação na junção entre a placa acrílica transparente e a placa acrílica leitosa.

\section{Ensaio de envelhecimento}

Foi feita uma primeira leitura colorimétrica nos corpos de prova. Posteriormente, os $24 \mathrm{CPs}$ foram adaptados no suporte metálico (Figura 3) e submetidos ao processo de envelhecimento acelerado, utilizando o equipamento Câmara de UV - Equilam ${ }^{\circledR}$ (Figura 4$)^{16}$ durante três semanas, totalizando 504 horas com ciclos de 4 horas com radiação UVA a 60 ${ }^{\circ} \mathrm{C}$, seguidos de 4 horas de condensação a $50{ }^{\circ} \mathrm{C}$, de acordo com as normas ASTM 154. O ensaio foi realizado no Laboratório de Corrosão do Instituto de Pesquisas Tecnológicas (IPT) de São Paulo, SP, Brasil. 


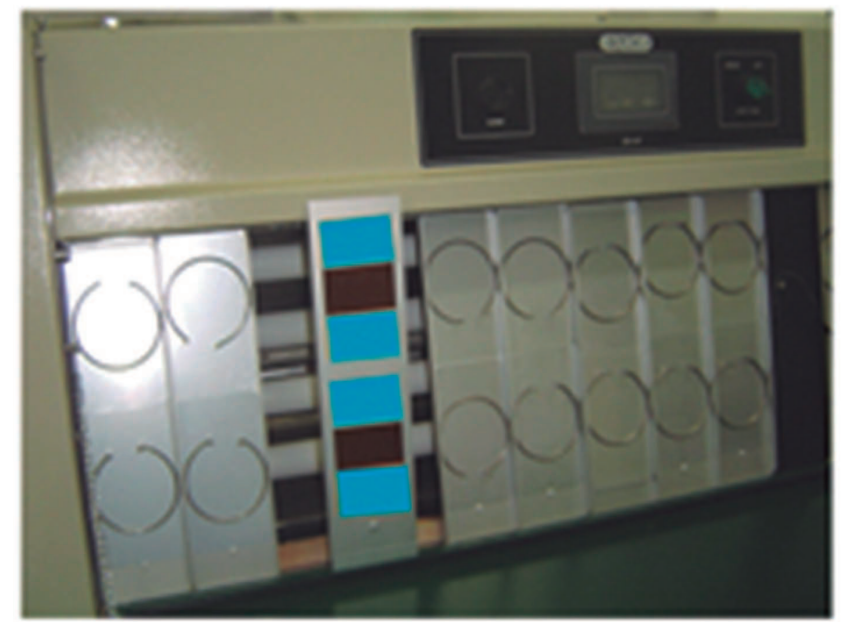

Figura 3 - Corpos-de-prova em posição para o ensaio

Foram realizadas novas leituras colorimétricas, utilizando o mesmo equipamento Minolta ${ }^{\circledR}$, na primeira, segunda e terceira semanas de ensaio de envelhecimento acelerado, sempre na mesma região de cada CP para evitar possíveis distorções, obtendo-se as medidas dos três coeficientes ( $\left.L^{*}, a^{*}, b^{*}\right)$ entre as leituras colorimétricas ${ }^{4}$.

\section{Análise estatística}

Os dados desta pesquisa foram processados pelo programa digital SPSS $12.0^{\circledR}$. A avaliação da fotodegradação de cor em função do tempo foi obtida por meio da Análise de Variância de Friedman (Friedman test) para três amostras dependentes, uma vez que os mesmos protótipos foram avaliados em três momentos diferentes.

A média do $\Delta \mathrm{E}^{*}$ entre os $4 \mathrm{CPs}$ da mesma cor foi utilizada como parâmetro para os testes, sendo considerados clinicamente aceitáveis os valores inferiores a 3,3, conforme norma presente em vários trabalhos com materiais odontológicos para restaurações estéticas ${ }^{6,8}$.

\section{Resultados}

Análises das diferenças de cor $\left(\Delta \mathrm{E}^{*}\right)$ em função do tempo após envelhecimento artificial

O comportamento na cor verde não apresentou diferença estatística entre os três períodos avalia$\operatorname{dos}(p=0,084)$, resultado obtido por meio do teste de Friedmam para mostras emparelhadas (Figura 4). $\mathrm{O} \Delta \mathrm{E}^{*}$ inicial foi de 0,90 ; na segunda semana, foi de 0,86 ; e o final para a cor verde apresentou o valor de 1,83 , abaixo do nível clinicamente aceitável, $\Delta \mathrm{E}^{*}$ 3,3 (Figura 4).

\begin{tabular}{|c|c|c|c|c|c|c|}
\hline & & & $\mathbf{N}$ & $x^{2}$ & gl & D \\
\hline \multirow{3}{*}{ Vorde } & semana 1 & 1,33 & & & & \\
\hline & semana 2 & 2,37 & 6 & 4,92 & 2 & D,0es \\
\hline & Semuna 3 & 2,50 & & & & \\
\hline \multirow{3}{*}{ Anul } & Semuna 1 & 2,83 & & & & \\
\hline & Semana 2 & 2,33 & 6 & 624 & 2 & 0,015 \\
\hline & Semuna 3 & 283 & & & & \\
\hline \multirow{3}{*}{ Morrom } & semans 1 & 1,67 & & & & \\
\hline & semuna 2 & 200 & 6 & 1,33 & 2 & Q.523 \\
\hline & Semana 3 & 2,33 & & & & \\
\hline \multirow{3}{*}{ Preto } & Semuna 1 & 200 & & & & \\
\hline & semuna 2 & 1,92 & 6 & 0,10 & 2 & Q953 \\
\hline & semana 3 & 2008 & & & & \\
\hline
\end{tabular}

Figura 4 - Teste de Friedman, amostras emparelhadas entre períodos

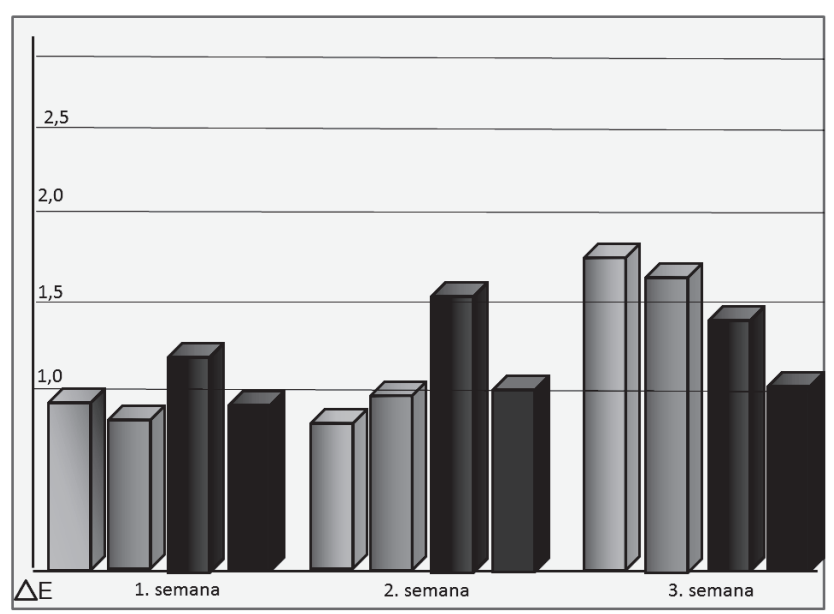

Figura 5 - Análise da variação média de $\left(\Delta E^{*}\right)$ das cores entre os períodos

Na cor azul, no grupo adesivo, o teste de Friedman mostrou diferença estatística entre os períodos avaliados, com valor do $\mathrm{p}=0,01$ (Tabela 1). O $\Delta \mathrm{E}^{*}$, inicialmente no valor de 0,79 , finalizou com o valor de 1,70, índice clinicamente aceitável, $\Delta \mathrm{E}^{*}=$ 3,3 (Figura 5).

$O$ valor de $p=0,513$ demonstra a não existência de diferença estatística da cor marrom (Tabela 1). A primeira leitura colorimétrica registrou $\Delta \mathrm{E}^{*}$ no valor de 1,18; na segunda semana, o valor foi de 1,61, finalizando em 1,56 (Figura 5).

$\mathrm{O}$ valor de $\Delta \mathrm{E}^{*}$ para a cor preta foi de 1,37 ; na segunda semana, foi de 1,40, finalizando em 1,06. Para a cor preta, não houve diferença estatística entre os períodos, $\mathrm{p}=0,953$ (Tabela 1 ).

Em nenhuma das cores houve uma degradação de cor acima do nível clinicamente aceitável.

\section{Discussão}

A fidelidade na reprodução da íris na confecção da prótese ocular é um fator fundamental no sucesso da reabilitação protética ${ }^{6,9,10}$. Outro fator im- 
portante está ligado à longevidade, que depende da fotodegradação causada pela exposição à radiação solar. Anteriormente, a fotografia era usada apenas como um meio para orientar a pintura da íris protética $^{5,10}$.

Na primeira publicação a respeito da utilização de imagem digital do olho contralateral impressa com jato de tinta, concluiu-se ser essa uma técnica que reduz o tempo de confecção, apresentando resultado estético satisfatório. Porém, estudos quanto ao fator fotodegradação, importantes para comprovar sua longevidade, não foram realizados ${ }^{5}$. $\mathrm{O}$ mesmo ocorre com trabalhos mais recentes, que descrevem novas técnicas, sem, no entanto, serem complementados com testes de envelhecimento ${ }^{11,12,13}$.

Outra nova técnica de confecção de prótese ocular envolveu a substituição da pintura tradicional da íris protética pela imagem digitalizada da íris remanescente, por meio de fotografia revelada em papel fotográfico, posteriormente submetida ao envelhecimento acelerado ${ }^{6}$. Quando comparada a pintura realizada tradicionalmente com tinta acrílica à técnica utilizando a imagem digital revelada em papel fotográfico nas cores azul, amarela, preta, marrom e verde, a degradação de cores mostrou uma semelhança entre os grupos pintura e fotografia. Nas cores verde e amarela, a alteração revelou-se um pouco acima do nível clinicamente aceitável. Para as cores marrom e preta, ocorreram diferenças significativas, com variações em níveis clinicamente aceitáveis; para a cor azul, em níveis elevados, inviáveis clinicamente tanto na fotografia como na pintura ${ }^{14}$.

Não foi encontrada, na literatura, pesquisa em que a íris protética digital tenha sido obtida com impressão em adesivo, como aqui se propõe.

Neste estudo, após a leitura colorimétrica, nenhuma das médias do $\Delta \mathrm{E}^{*}$ das cores avaliadas finalizou com alteração acima do nível clinicamente aceitável, $\Delta \mathrm{E}^{*}=3,3$. O maior valor foi de 1,83 para a cor verde, e o menor valor, de 1,06 para a cor preta. Essa baixa degradação na cor verde também foi encontrada em outras pesquisas ${ }^{6,15,16}$.

Na cor azul, o teste de Friedman mostrou a única diferença estatística entre os períodos avaliados neste estudo, o valor do $\mathrm{p}=0,015$. Para a cor azul, na primeira semana, os valores de $\Delta \mathrm{E}^{*}$ ficaram em 0,79 ; na segunda semana, os valores médios de $\Delta \mathrm{E}^{*}$ foram de 0,96; e, na terceira semana, o valor de $\Delta \mathrm{E}^{*}$ foi de 1,70, índice clinicamente aceitável. Em outros estudos, a degradação da cor azul foi acima do clinicamente aceitável. Na pesquisa em que se comparou o grupo fotografia digital com o grupo pintura, a cor azul apresentou diferenças estatísticas nos três períodos e uma importante degradação final em ambos os grupos, bem maior que em outras cores, com valores de $\Delta \mathrm{E}=26,30$ na fotografia e de 24,68 na pintura ${ }^{6}$. Outra expressiva degradação da cor azul ocorreu quando foi comparada a técnica digital à da pintura com tinta a óleo. Avaliando o comportamento antes e após a polimerização, sem ensaio de envelhecimento acelerado, a cor azul apresentou $\Delta \mathrm{E}^{*}=20,23$ para a tinta a óleo e 16,61 para a técnica digital ${ }^{16}$. Em estudos com tinta acrílica hidrossolúvel e tinta automotiva laca acrílica, obteve-se, para o pigmento azul, índices elevados de alteração de cor $^{12}$. Da mesma forma, Fernandes et al. ${ }^{17}$, em 2005, avaliando próteses oculares polimerizadas em micro-ondas, constataram comportamento de instabilidade para o pigmento azul nos cinco tipos de tinta avaliados. Estudos comparando as cores marrom e azul obtiveram os seguintes valores de $\Delta \mathrm{E}^{*}$ para a cor azul: tinta óleo, 6,22 ; tinta acrílica, 7,83 ; tinta guache, 5,96 ; e pigmentos de resina para caracterização da esclera, 6,5017. Quando a degradação de cor azul da porção escleral empregada na caracterização da prótese ocular foi estudada, verificou-se uma estabilidade muito aquém do limite clinicamente aceitável, com $\Delta \mathrm{E}^{*}$ em 21,93 e 21,68 ${ }^{18}$.

Para a cor marrom, a primeira leitura colorimétrica registrou $\Delta \mathrm{E}^{*}$ no valor de 1,18 ; na segunda semana, o valor foi de 1,61, finalizando em 1,56. Valores baixos de $\Delta \mathrm{E}^{*}$ foram, também, obtidos em outro estudo, com 2,91 no grupo fotografia digital e 1,53 no grupo pintura ${ }^{6,19}$. Pesquisa realizada, comparando tinta automotiva com tinta acrílica, ambos os grupos apresentaram uma degradaçaõ acima do limite clínico aceitável ${ }^{19}$. Em outro estudo observou-se uma forte degradação na cor marrom no grupo pintura no valor de 16,68 e na técnica digital $20,23^{16}$.

$\mathrm{Na}$ cor preta, não houve diferença estatística entre os períodos $\mathrm{p}=0,953$, e os valores de $\Delta \mathrm{E}^{*}$ foram de 1,37 na primeira semana, de 1,40 na segunda e de 1,06 na terceira. Estudo comparando a imagem digital com a pintura apresentou, para a cor preta, índice de degradação de 3,00 para a fotografia e 2,04 para a pintura ${ }^{6}$. Índices baixos de degradação para a cor preta também foram encontrados em outros estudos ${ }^{19}$.

\section{Conclusão}

A técnica proposta neste estudo mostrou-se eficaz na fidelidade de reprodução da íris remanescente pelo método digital e na diminuição do tempo de confecção das próteses oculares, apresentando, ainda, baixo custo, boa longevidade e possibilidade de armazenamento das imagens para eventuais trocas da prótese. 


\section{Abstract}

Introduction: one of the essential factors in satisfactory final result of ocular prosthetic rehabilitation treatment is a faithful reproduction of the iris of the eye remaining in prosthetic iris. It concealment loss for affected patients. Objective: this paper presents a new technique to manufacture prosthetic iris, using the remaining iris scanned image, obtained by photographic image printed on adhesive. Methods: depending on the photo degradation that compromises the longevity of artificial eyes, decided to evaluate the stability of colors, comparing both techniques, through accelerated aging test. For the tests were prepared specimens simulating prosthetic iris, divided into two groups: adhesive sticker and, in green, blue, brown and black. The variation of the degree of degradation was evaluated by colorimetric reading before and after and three weekly intervals during aging with artificial ultraviolet radiation. Statistical analysis suggests that when the colors were evaluated according to the period of artificial aging test, only in blue color in the adhesive group difference was statistically significant. Results: when evaluated as a function of group and trial period, the statistical tests indicate no difference between the colors of adhesive. Conclusion: none of the colors between the groups showed color degradation above the clinically acceptable level.

Keywords: Artificial eye. Iris. Colour.

\section{Referências}

1. Goulart DR, Queiroz E, Fernandes AÚ, Oliveira LM Psychosocial aspects in the rehabilitation of patients with anophthalmic socket: implications of the use of ocular prosthesis. Arq Bras Oftalmol 2011; 74(5):330-4.

2. Rezende JRV. Fundamentos da prótese buco maxilo facial. São Paulo: Sarvier; 1997. p. 129-44.

3. Goiato MC, Fernandes AÚ, dos Santos DM, Hadadd MF, Moreno A, Pesqueira AA. Alteration of blue pigment in artificial iris in ocular prosthesis: effect of paint, drying method and artificial aging. Cont Lens Anterior Eye 2011; 34(1):22-5.

4. Brennan P, Fedor C. Sunlight UV, \& accelerated weathering Technical Bulletin LU-0822. The Q-Panel Lab Products; 1994. LU-0822. p. 1-7.

5. Artopolou II, Montgomery PC, Wesley PJ, Lemon JC. Digital imaging in the fabrication of ocular prostheses. J Prosthet Dent 2006; 95(4):327-30.

6. Reis RC. Avaliação da íris de prótese ocular obtida através de imagem digitalizada impressa em papel fotográfico [Dissertação de Mestrado]. São Paulo: Universidade de São Paulo, Faculdade de Odontologia; 2008.

7. Ramalho J. Fotografia digital. 3. ed. Rio de Janeiro: Elsevier; 2004.

8. Dias RB, Rezende JRV, Carvalho JC. Light-weight ocular prosthesis. Braz Dent J 1994; 5(2):105-8.

9. Jain S, Makkar S, Gupta S, Bhargava A. Prosthetic rehabilitation of ocular defect using digital photography: a case report. J Indian Prosthodont Soc 2010; 10(3):190-3.
10. Murphey PJ, Pitton RD, Schlossberg L, Harris LW. The development of acrylic eye prosthesis at The National Naval Medical Center. J Am Dent Assoc 1945;32(19): 1227-44.

11. Jayaswal GP, Dange SP, Khalikar AN.Restoration of an atrophic eye socket with custom made eye prosthesis, utilizing digital photography. Indian J Dent Res 2011; 22(3):4825 .

12. Sidharth J, Sumit M, Sharad G. Akashay bhargava prosthetic rehabilitation of ocular defect using digital photography: a case report. J Indian Prosthodontic Soc 2010; 10(3):190-3.

13. Kale $\mathrm{E}, \mathrm{Me} \sqcap \mathrm{A}$, Izgi $\mathrm{AD}$. A technique for fabrication of an interim ocular prosthesis. J Prosthodont 2008; 17(8):654-61.

14. Reis RC, Dias RB, Carvalho JCM. Evaluation of iris color stability in ocular prosthesis. Braz Dent J 2008; 19:370-4.

15. D’Almeida NF. Avaliação da estabilidade de cor das tintas empregadas na pintura de íris em prótese ocular [Dissertação de Mestrado]. São José dos Campos: Faculdade de Odontologia da Unesp; 1999.

16. Goiato MC, dos Santos DM, Moreno A, Gennari-Filho H, Pellizzer EP. Evaluation of the color stability of two techniques for reproducing artificial irides after microwave polymerization. J Appl Oral Sci 2011; 19(3):200-3.

17. Fernandes AU, Goiato MC, Batista MA, Santos DM. Color alteration of the paint used for iris painting in ocular prostheses. Braz Oral Res 2009; 23(4):386-92.

18. Kabawata SC. Avaliação da estabilidade de cor da esclera em prótese ocular [Dissertação de Mestrado]. São Paulo: Universidade de São Paulo, Faculdade de Odontologia; 2006.

19. Mundim FM, Antunes PL, Sousa AB, Garcia LF, Pires-de-Souza FC. Influence of artificial accelerated ageing on the colour stability of paints used for ocular prosthesis iris apainting. Gerodontology 2011; 29(2):312-7.

\section{Endereço para correspondência:}

Ricardo Cesar dos Reis

Avenida Padre Antonio José dos Santos, 530 apto. 163, Brooklin 04563-001 São Paulo - SP

Fones: (11) 55431861, cel. (11) 99988-4699

E-mail: rcreis@usp.br

Recebido: 04/03/2013. Aceito: 01/08/2013. 